\title{
Neuroticism and the Overlap Between Autistic and ADHD Traits: Findings From a Population Sample of Young Adult Australian Twins
}

\author{
Shin-Ho Park, ${ }^{1}$ Adam J. Guastella, ${ }^{1}$ Michael Lynskey, ${ }^{2}$ Arpana Agrawal, ${ }^{3}$ John N. Constantino, ${ }^{3}$ \\ Sarah E. Medland, ${ }^{4}$ Yun Ju C. Song, ${ }^{1}$ Nicholas G. Martin, ${ }^{4}$ and Lucía Colodro-Conde ${ }^{4}$ \\ ${ }^{1}$ Autism Clinic for Translational Research, Brain and Mind Centre, Central Clinical School, Faculty of Medicine, University of \\ Sydney, Sydney, NSW, Australia \\ ${ }^{2}$ Institute of Psychiatry, Psychology, and Neuroscience, King's College London, London, UK \\ ${ }^{3}$ Department of Psychiatry, Washington University School of Medicine, St. Louis, MO, USA \\ ${ }^{4}$ Genetics \& Computational Biology Department, OIMR Berghofer Medical Research Institute, Brisbane, Queensland, \\ Australia
}

Neuroticism, a 'Big Five' personality trait, has been associated with sub-clinical traits of both autism spectrum disorder (ASD) and attention deficit hyperactivity disorder (ADHD). The objective of the current study was to examine whether causal overlap between ASD and ADHD traits can be accounted for by genetic and environmental risk factors that are shared with neuroticism. We performed twin-based structural equation modeling using self-report data from 12 items of the Neo Five-Factor Inventory Neuroticism domain, 11 Social Responsiveness Scale items, and 12 Adult ADHD Self-Report Scale items obtained from 3,170 young adult Australian individual twins (1,081 complete pairs). Univariate analysis for neuroticism, ASD, and ADHD traits suggested that the most parsimonious models were those with additive genetic and unique environmental components, without sex limitation effects. Heritability of neuroticism, ASD, and ADHD traits, as measured by these methods, was moderate (between $40 \%$ and $45 \%$ for each respective trait). In a trivariate model, we observed moderate phenotypic (between 0.45 and 0.62), genetic (between 0.56 and 0.71 ), and unique environmental correlations (between 0.37and 0.55) among neuroticism, ASD, and ADHD traits, with the highest value for the shared genetic influence between neuroticism and self-reported ASD traits $\left(r_{\mathrm{g}}=\right.$ 0.71). Together, our results suggest that in young adults, genetic, and unique environmental risk factors indexed by neuroticism overlap with those that are shared by ASD and ADHD.

Keywords: neuroticism, autism, ADHD, twin study

Autism spectrum disorder (ASD) and attention deficit/ hyperactivity disorder (ADHD) are neurodevelopmental conditions characterized by complex heterogeneous presentations and unknown cause. ASD is principally characterized by impaired social interaction and communication, and restrictive and repetitive behavior. ADHD is defined by symptoms of inattention, impulsivity, and hyperactivity (Diagnostic and Statistical Manual 5 [DSM-5]; American Psychiatric Association, 2013). During the last two decades, the prevalence rate in children and adults with ASD or ADHD has dramatically risen. ASD is currently estimated to influence 1 in 68 children (Centers for Disease Control Prevention, 2014) and 1 in 100 adults (Brugha et al., 2011). For ADHD, more than 1 in 10 children (Visser et al., 2014) and 1 in 30 adults (Fayyad et al., 2007) are estimated to be affected. Both disorders are heritable, with heritability from external ratings ranging between $80 \%$ and $90 \%$ for ASD (Lichtenstein et al., 2010; Losh et al., 2009) and about 80\% for ADHD (Faraone et al., 2005; Lichtenstein et al., 2010) in children. In contrast, the heritability estimates of self-rated ASD and ADHD traits are about 57\% (Hoekstra et al., 2007) and 30-40\% (Boomsma et al., 2010; Larsson et al., 2013; Reiersen et al., 2008; van den Berg et al., 2006), respectively, in adult samples.

RECEIVED 7 May 2017; ACCEPTED 30 May 2017

ADDRESS FOR CORRESPONDENCE: Dr Lucía Colodro Conde, Genetics \& Computational Biology Department, QIMR Berghofer Medical Research Institute, 300 Herston Road, Brisbane, QLD 4006, Australia. E-mail: Lucia.ColodroConde@qimrberghofer. edu.au 
Studies have further reported the frequent cooccurrence of ASD and ADHD (Rommelse et al., 2010). Approximately, $28-44 \%$ of the adult population diagnosed with ASD also meets Diagnostic and Statistical Manual $I V(D S M-I V)$ criteria (American Psychiatric Association, 2000) for ADHD (Johnston et al., 2013; Lai et al. 2014). While ASD and ADHD negatively impact on daily functioning, this impact is particularly severe when these conditions occur together (Rao \& Landa, 2014). The estimated annual economic cost for individuals with ASD and ADHD were U.S. $\$ 268$ billion (Leigh \& Du, 2015) and U.S. \$143-266 billion (Doshi et al., 2012) in the United States, respectively.

Despite the high rates of comorbidity between ASD and ADHD, the underlying cause of their co-occurrence remains poorly understood. So far, there have been only four adult twin studies investigating the etiology of co-occurrence of ASD and ADHD traits. Two studies (Lundstrom et al., 2011; Reiersen et al., 2008), suggested that the co-occurrence of these disorders might be due to substantial genetic overlap between these two traits (genetic correlation; $r_{\mathrm{g}}=\sim 0.60$ ). Polderman and colleagues, further investigated the association between ASD and ADHD traits using the five trait-based dimensional measures of ASD (social skills, routine, attentional switching, imagination, and patterns) and two dimensions of ADHD (inattention and hyperactive/impulsive problems). Their study reported that this association would be the result of attention-related problems (Polderman et al., 2013). Another study conducted by the same author (Polderman et al., 2014) reported a substantial genetic overlap between restrictive-repetitive behaviors/interests and ADHD dimensions $\left(r_{\mathrm{g}}=0.61-0.64\right)$ using measures based on the DSM-5; however, the genetic overlap between social communication difficulties and ADHD dimensions was weak $\left(r_{\mathrm{g}}=0.20-0.22\right)$. Although our understanding of the genetic structure of these disorders is changing rapidly, molecular studies have failed to show shared genetic etiology for ADHD and ASD, reflecting (a) lack of power to detect common associated variants of small effect (Bulik-Sullivan et al., 2015; Cross-Disorder Group of the Psychiatric Genomics Consortium, 2013), and possibly (b) the confounding effects of unmeasured cross-traits in case-control study designs, that is, failure to control for ADHD traits in comparison groups in molecular genetic studies of ASD, and conversely, failure to control for ASD traits in comparison groups in molecular genetic studies of ADHD, which the effect of either would be to erode the ability to detect overlapping genetic variance.

Increasing evidence suggests that the continua of behavioral traits, whose extremes reflect clinical neuropsychiatric disorders are contributed to by variations in what has historically been construed as personality, and that the symptom structure of psychopathology in general may be more parsimonious than has been traditionally appreciated.
Specifically, neuroticism, defined as the tendency to experience negative emotions (Matthews et al., 2003), may share causal origins with the drivers of comorbidity between ASD and ADHD. Neuroticism is a moderately heritable personality trait with heritability estimates ranging from $49 \%$ to 56\% (Hansell et al., 2012; Kendler et al., 2007) and characterized by proneness to negative emotions, such as anxiety, sadness, guilt, fear, anger, and stress (Matthews et al., 2003; Zuckerman \& Stelmack, 2004). Some of these characteristics are noted as frequently elevated in both ASD (Duvekot et al, 2016; Gillott et al., 2001; Gillott \& Standen, 2007; Hurtig et al., 2009) and ADHD (Alexander \& Harrison, 2013; Harrison et al., 2013) population. Moreover, clinicbased (Schriber et al., 2014; Schwartzman et al., 2016) and population-based (Austin, 2005; Wakabayashi et al., 2006) studies have indicated that higher neuroticism is associated with higher levels of autistic traits. Several lines of evidence also suggest that higher neuroticism is linked with the severity of ADHD (Jacob et al., 2007; Knouse et al., 2013; Michielsen et al., 2014; Nigg et al., 2002; Parker et al., 2004). The co-occurrence of ASD and anxiety (Duvekot et al., 2016) and ASD and ADHD (Musser et al., 2014; van Steijn et al., 2012) may be partly explained by their shared familial transmission, indicating overlap in familial risk. On the whole, neuroticism is the only Big Five trait to be consistently associated with both ASD and ADHD traits, while conscientiousness, agreeableness, and openness to experience showed inconsistent results. Moreover, neuroticism (or negative emotionality) has been proposed to represent a non-specific vulnerability factor that underlies and shares genetic overlap with multiple dimensions of psychopathology (Tackett, et al., 2013). Neuroticism could represent an example of general predisposition to psychopathology, influencing the development and severity of ASD, ADHD, and other psychological and psychiatric outcomes (Constantino, 2017) and so be a non-trait or non-diagnostic specific susceptibility factor for general psychopathology. At present, molecular studies have shown no evidence of pleiotropy between neuroticism and the ADHD (Gale et al., 2016) - and there is no data available about neuroticism and ASD. The contribution of genetic and environmental factors to the comorbidity of neuroticism, ASD, and ADHD has not been investigated.

A previous study conducted with a sample partially overlapping with the one used in the present study (Reiersen et al., 2008) suggested that in young adults, a substantial overlap of the genetic influences on autistic traits and self-reported inattention symptoms of ADHD may lead to their frequent co-occurrence. However, this study was conducted in a small sample size $(N=674)$. Even though they were not detected, we were unable to draw conclusions about common environmental effects, genetic dominance, sex-limitation effects, and sibling-interaction effects because of limited power. In addition to this, it is still unclear whether these findings on the high genetic correlation 
between self-rated ASD and ADHD traits $\left(r_{\mathrm{g}}=0.72\right)$ is largely driven by neuroticism.

The aim of the present study was to investigate the relationship between neuroticism, ASD, and ADHD traits (inattentive and impulsive symptoms). Using a large sample $(N=3,170)$, we sought to: (1) replicate our prior work (Reiersen et al., 2008) showing the evidence for substantial genetic correlation between ASD and ADHD traits, using a larger sample; and (2) examine the degree to which the co-occurrence between ASD and ADHD traits could be explained by shared genetic and environmental risk factors in common with neuroticism.

\section{Materials and Methods}

\section{Subjects and Procedure}

The data reported here included an unselected populationbased sample of 3,202 twins born between 1962 and 1987 enrolled in the Australian Twin Registry as part of a study focused on cannabis and other substance use (Lynskey et al., 2002).

After excluding individual participants with missing items $(n=10)$ or unknown zygosity $(n=22)$, the sample comprised a total of 3,170 twins (1,081 complete pairs), including 371 monozygotic (MZ) female, $154 \mathrm{MZ}$ male, 278 dizygotic (DZ) female, 95 DZ males, and 183 opposite-sex (OS) twin pairs. However, single twins from incomplete pairs were used in the Full Information Maximum Likelihood (FIML) analysis to better estimate the sample distribution of means, variances, and thresholds. Zygosity was ascertained by questionnaire. Mean age at completion was $32.31(S D=2.5)$. Informed consent was obtained from participants. This study was approved by the QIMR Berghofer Human Research Ethics Committee, the Institutional Review Board at Washington University School of Medicine, and the Australian Twin Registry Ethics Committee.

\section{Measures}

Participants completed an online or paper-and-pencil survey on health and well-being, including the NEO FiveFactor Inventory (NEO-FFI; Costa \& McCrae, 1992), for the measure of personality, a modified short version of the Social Responsiveness Scale (SRS; Constantino \& Gruber, 2005), and the Adult ADHD Self-Report Scale (ASRS; Kessler et al., 2005), which were designed to minimize subject burden and improve data quality.

Neo Five-Factor Inventory-Neuroticism (NEO-FFI-N). Neuroticism was measured by summing 12 items of the Neuroticism domain of the short form of the Neo-FFI, which consists of a total of 60 items (Costa \& McCrae, 1992). Each NEO-FFI-N item was scored on a 5-point scale $(0=$ strongly disagree $; 1=$ disagree $; 2=$ neutral $; 3=$ agree $;$ $4=$ strongly agree) with total scores ranging from 0 to 48 .
Cronbach's alpha for this scale was 0.89 , indicating high inter-item reliability.

The Social Responsiveness Scale (SRS). The SRS (Constantino \& Gruber, 2005) is a 65-item questionnaire based on the DSM-IV and designed to assess autistic traits. Each SRS item is scored on a 4 -point scale $(0=$ false, not at all true; $1=$ slightly true; $2=$ mainly true; $3=$ very true). In the present study, the total number of items was limited to 11 items, with total sum scores ranging from 0 to 33 . The modified version of SRS included the items that measure the autistic symptoms the three DSM-IV autism domains: Social Cognition (SRS items 15, 42, and 58), Social Communication (SRS items 16, 18, 35, and 37), Social Motivation (SRS item 6), and Autistic Mannerisms (SRS items 24, 29, and 39). These 11 items had strong loadings on the first unrotated factor of a principal components analysis of the SRS in a pediatric sample (Constantino et al., 2000). Cronbach's alpha for SRS was 0.83 , indicating high inter-item reliability at least with child psychiatric patients.

Adult ADHD Self-Report Scale (ASRS). The ASRS (Kessler et al., 2005) is a widely used short screening tool for ADHD. It includes 18 items that references the DSM-IV ADHD criteria (American Psychiatric Association, 2000). The modified version of ADHD included the 12 items that seemed most applicable to this age group ( 9 inattentive and 3 impulsive symptoms). We did not include the ASRS items related to the DSM-IV hyperactive symptoms, because these symptoms tended to have less stability than inattentive symptoms in this age group (Todd et al., 2008). Similar to SRS items, each ASRS item is scored on a 4-point scale $(0=$ false, not at all true $; 1=$ slightly true $; 2=$ mainly true; $3=$ very true) with total sum scores ranging from 0 to 36 . This questionnaire also demonstrated good internal consistency (Cronbach's alpha $=0.84$ ).

Total raw scores were calculated for each questionnaire by summing the scores of the relevant items. Higher scores indicated greater severity. Examination of the distribution revealed that the SRS and ASRS data were highly skewed (skewness $=1.97$ for SRS and 1.22. for ASRS). Hence, we used a threshold model under the assumption of an underlying continuous liability distribution with the thresholds defining categories. A simulation study by Derks and colleagues (2004) showed that this is the best approach when analyzing L-shaped distributed phenotypic data as it produced unbiased parameter estimates. Otherwise, the skewness in the data leads to biases in parameter estimates, including underestimation of the shared environmental factors and overestimation of the unique environmental factors.

These continuous scores were clustered in rank-ordered groups of individuals. The number of categories was chosen in such way that the number of subjects in each category was roughly similar, in order to avoid small 

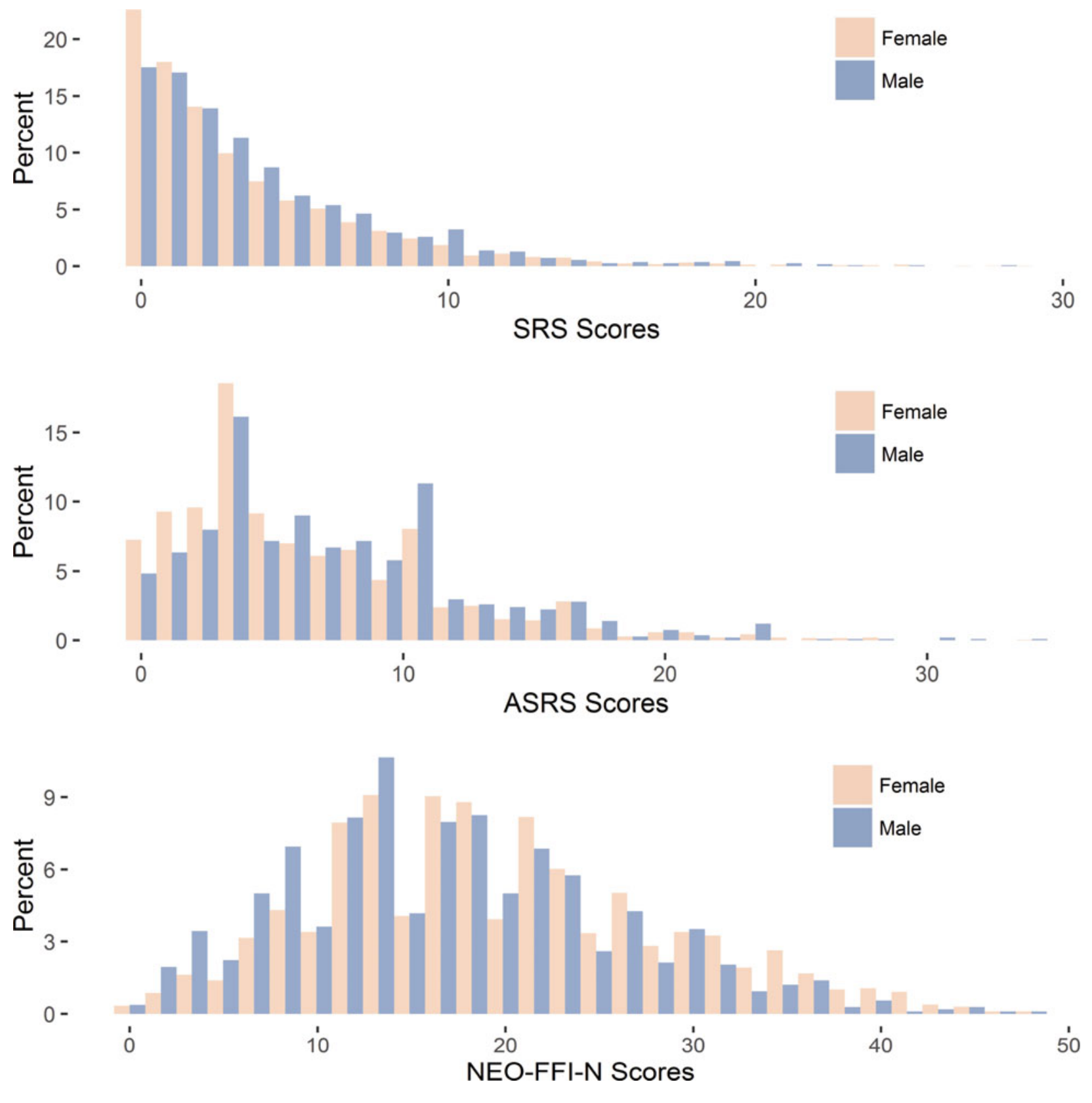

FIGURE 1.

(Colour online) Distribution of raw SRS, ASRS, and neuroticism scores by sex.

cell size. NEO-FFI-N scores had only modest skew (skewness $=0.44)$ and, therefore, did not require transformation. Figure 1 shows the distributions by sex for the three variables.

\section{Statistical Analyses}

Initially, a saturated model in which all the parameters are allowed to be free was used to test hypotheses concerning means, variances (or thresholds), and covariance in the analysis of individual observations for twin pairs for the three variables (Neale \& Cardon, 1992).
The results from the saturated models were used in designing a univariate modeling. In the present study, we applied the classical twin design (Neale \& Cardon, 1992) and implemented structural equation modeling using OpenMx (Boker et al., 2011) to estimate the amount of the variances in scores due to additive genetic (A), shared environmental $(C)$, dominant genetic (D), and unique environmental (E) factors. However, these components cannot be estimated simultaneously, since dominant genetic and shared environmental components are negatively confounded (Martin et al., 1978). Therefore, we chose to model either an ACE or an ADE, based on the pattern of twin correlations. MZ 


\section{TABLE 1}

Twin Correlations for Different Zygosity Groups and Total MZ and DZ Pairs $(95 \% \mathrm{Cl})$

\begin{tabular}{lllll}
\hline Zygosity & Complete pairs & NEO-FFI-N & SRS & ASRS \\
\hline MZ females & 371 & $0.37[0.28,0.45]$ & $0.40[0.29,0.49]$ & $0.38[0.28,0.47]$ \\
DZ females & 278 & $0.27[0.17,0.37]$ & $0.29[0.16,0.42]$ & $0.30[0.16,0.42]$ \\
MZ males & 154 & $0.41[0.26,0.53]$ & $0.50[0.35,0.63]$ & $0.46[0.31,0.59]$ \\
DZ males & 95 & $0.17[-0.03,0.35]$ & $0.33[0.11,0.51]$ & $0.02[-0.21,0.24]$ \\
DZ opposite sex & 183 & $0.16[0.02,0.29]$ & $0.23[0.06,0.38]$ & $0.23[0.06,0.38]$ \\
All MZ pairs & 525 & $0.38[0.30,0.45]$ & $0.43[0.34,0.50]$ & $0.40[0.32,0.48]$ \\
All DZ pairs & 556 & $0.22[0.14,0.30]$ & $0.28[0.19,0.36]$ & $0.23[0.13,0.32]$ \\
\hline Note: NEO-FFI-N = Neo Five-Factor Inventory-Neuroticism; SRS = Social Responsiveness Scale; ASRS = Adult ADHD Self- \\
\multicolumn{2}{r}{ Report Scale. }
\end{tabular}

twins share $100 \%$ of their genes, and DZ twins share $50 \%$ of their genes. If the $\mathrm{MZ}$ correlation is more, than twice the DZ twin correlation, we used the ADE model as the MZ correlation indicates the presence of the genetic effects due to dominance over additive genetic influences. For $\mathrm{MZ}$ correlations less than twice the $\mathrm{DZ}$ correlations, we used the ACE model as the presence of both $\mathrm{A}$ and $\mathrm{C}$ components are indicated.

We obtained the nested sub-models by dropping A or C (or D) or both parameters. The fit of each sub-model was assessed by the difference in log likelihood between the sub and full models. If sub-model fit was significantly worse than the full model, the full model was accepted. Otherwise, the sub-model was used as the most parsimonious model.

Next, a trivariate Cholesky decomposition model was fitted to estimate the magnitude of genetic and environmental influences that mediate the phenotypic covariation between NEO-FFI-N, SRS, and ASRS scores. The choice of parameters for this trivariate Cholesky decomposition model was based on the results from the univariate modeling.

\section{Results}

First, a saturated model was used to check whether means and variances or thresholds are equal across different zygosity groups, and for the presence of genetic and sexlimitation effects as well as age and sex effect differences. No differences in means, variances, or thresholds were found across birth order and zygosity in any of the variables, except for NEO-FFI-N, where there was an apparent difference of means across like-sex MZ (24.8 for males, 20.8 for females) and DZ twins (23.6 for males and 22.8 for females) in the assumption testing $\left(\Delta \chi^{2}=9.49, \Delta d f=2, p<.009\right)$. In the structural equation modeling, we allowed the means to vary across zygosity groups ( $\mathrm{MZ}$ and $\mathrm{DZ}$, including opposite sex twins) in the full model and tested if there was a significant difference of fit with a model where the means were equated. Since there were no significant differences, we assumed equal means across zygosity for the rest of the analyses. For SRS and ASRS, difference in thresholds were found for $\operatorname{sex}\left(\Delta \chi^{2}=15.47,25.35, \Delta d f=3, p<.001\right)$. Therefore, sex was modeled as a covariate for these two variables. In addition, the effect of age was also controlled on
SRS $\left(\Delta \chi^{2}=6.58, \Delta d f=1, p<.01\right)$. There was a significant difference $\left(\Delta \chi^{2}=78.82,80.51,74.01, \Delta d f=1, p=<.001\right.$, $<.001,<.001)$, when we constrained all twin covariances to zero, indicating for all three variables the presence of familial aggregation. For all three variables, we found significant differences in $\mathrm{MZ}$ and $\mathrm{DZ}$ twin correlations $\left(\Delta \chi^{2}=8.99\right.$, $6.03,7.95, \Delta d f=1, p<.003,<.01,<.005)$, indicating that NEO-FFI-N, SRS, and ASRS are influenced by genetic factors. There was no indication for a sex-limitation model for any of the variables.

FIML estimates of twin correlations are presented in Table 1. The correlations between MZ twins are significantly greater than $\mathrm{DZ}$ twins (see above). However, the $\mathrm{MZ}$ correlations were less than twice as high as the DZ correlations, suggesting that the covariance between them might be influenced by genes and by shared environmental influences. Thus, an ACE model was fitted to the data in two zygosity groups (MZ and DZ).

We fitted univariate models to disentangle the sources of variance of the three phenotypes. As shown in Table 2, our univariate analysis for each score suggested that the most parsimonious univariate models were those with the additive genetic (A) and environmental (E) components. Additive genetic factors accounted for $45 \%(95 \%$ CI $[37,51])$ of the variance of SRS scores, $41 \%$ (95\% CIs $[33,48])$ of ASRS scores, and $41 \%(95 \%$ CI $[34,47])$ of the variance of NEOFFI-N scores, whereas unique environmental influences accounted for the remaining variance in each score. The common environmental factor $(\mathrm{C})$ could be dropped from our ACE model with no significant deterioration in fit. Hence, we only included A and E in our trivariate Cholesky decomposition model (Figure 2).

Figure 2 shows the parameter estimates from the trivariate Cholesky decomposition model with standardized path coefficients. Following the order of the variables, a common genetic factor shared (A1) accounted for all the genetic variance ( $41 \%$ of the total variance) in NEO-FFI-N, half of the genetic variance ( $22 \%$ of the total variance) in SRS, and onethird of the genetic variance ( $13 \%$ of the total variance) in ASRS. A further $50 \%$ of the genetic variance $(22 \%$ of the total variance) in SRS and $10 \%$ of the genetic variance ( $4 \%$ of the total variance) in ASRS can be explained by the genetic factor (A2) shared with SRS and ASRS. The genetic 
TABLE 2

Model-Fitting Results for the Best Fitting Univariate Models

\begin{tabular}{|c|c|c|c|c|c|c|c|c|c|}
\hline Model & $-2 \mathrm{LL}$ & $d f$ & $\Delta-2 \mathrm{LL}$ & $\Delta d f$ & $p$ & $\mathrm{AlC}$ & $A$ & $C / D$ & $E$ \\
\hline \multicolumn{10}{|c|}{ NEO-FFI-N } \\
\hline ACE & $15,590.51$ & 2,172 & 0.39 & 1 & .535 & $11,246.51$ & $0.33[0.13,0.47]$ & $0.06[0.00,0.22]$ & $0.60[0.53,0.67]$ \\
\hline $\mathrm{AE}$ & $15,591.01$ & 2,173 & 0.50 & 1 & .479 & $11,245.01$ & $0.41[0.34,0.47]$ & 0 & $0.59[0.53,0.66]$ \\
\hline CE & $15,601.17$ & 2,173 & 10.66 & 1 & .001 & $11,255.17$ & 0 & $0.31[0.26,0.37]$ & $0.69[0.63,0.74]$ \\
\hline $\mathrm{E}$ & $15,713.01$ & 2,174 & 122.50 & 2 & $<.001$ & $11,365.01$ & 0 & 0 & 1 \\
\hline \multicolumn{10}{|l|}{ SRS } \\
\hline ACE & $5,832.21$ & 2,168 & & & & $1,496.406$ & $0.29[0.05,0.50]$ & $0.13(0.00,0.32]$ & $0.57(0.50,0.66]$ \\
\hline $\mathrm{AE}$ & $5,834.21$ & 2,169 & 1.80 & 1 & .179 & $1,496.214$ & $0.45[0.37,0.51]$ & 0 & $0.55(0.49,0.63]$ \\
\hline CE & $5,838.19$ & 2,169 & 5.79 & 1 & .016 & $1,500.194$ & 0 & $0.35(0.29,0.41]$ & $0.65(0.59,0.71]$ \\
\hline $\begin{array}{l}\mathrm{E} \\
\text { ASRS }\end{array}$ & $5,945.99$ & 2,170 & 113.58 & 2 & $<.001$ & $1,605.987$ & 0 & 0 & 1 \\
\hline $\mathrm{ACE}$ & $5,912.68$ & 2,169 & & & & $1,574.678$ & $0.37[0.12,0.48]$ & $0.04[0.00,0.24]$ & $0.60[0.52,0.68]$ \\
\hline $\mathrm{AE}$ & $5,912.82$ & 2,170 & 0.14 & 1 & .71 & $1,572.815$ & $0.41[0.33,0.48]$ & 0 & $0.59[0.52,0.67]$ \\
\hline CE & $5,921.17$ & 2,170 & 8.49 & 1 & .004 & $1,581.171$ & 0 & $0.32[0.25,0.38]$ & $0.68[0.62,0.75]$ \\
\hline$E$ & $6,006.54$ & 2,171 & 93.86 & 2 & $<.001$ & $1,664.51$ & 0 & 0 & 1 \\
\hline
\end{tabular}

Note: Bold values indicate best-fitting models. $\mathrm{A}=$ additive genetic factors; $\mathrm{C}=$ common environmental factors; $\mathrm{E}=$ unique environmental factors; $-2 \mathrm{LL}=$ twice negative log-likelihood; $\Delta-2 \mathrm{LL}=$ difference in $-2 \mathrm{LL} ; d f=$ degrees of freedom; $\Delta d f=$ difference in degrees of freedom. Models compared with either the ACE or ADE model where the model with the smallest AIC value selected.

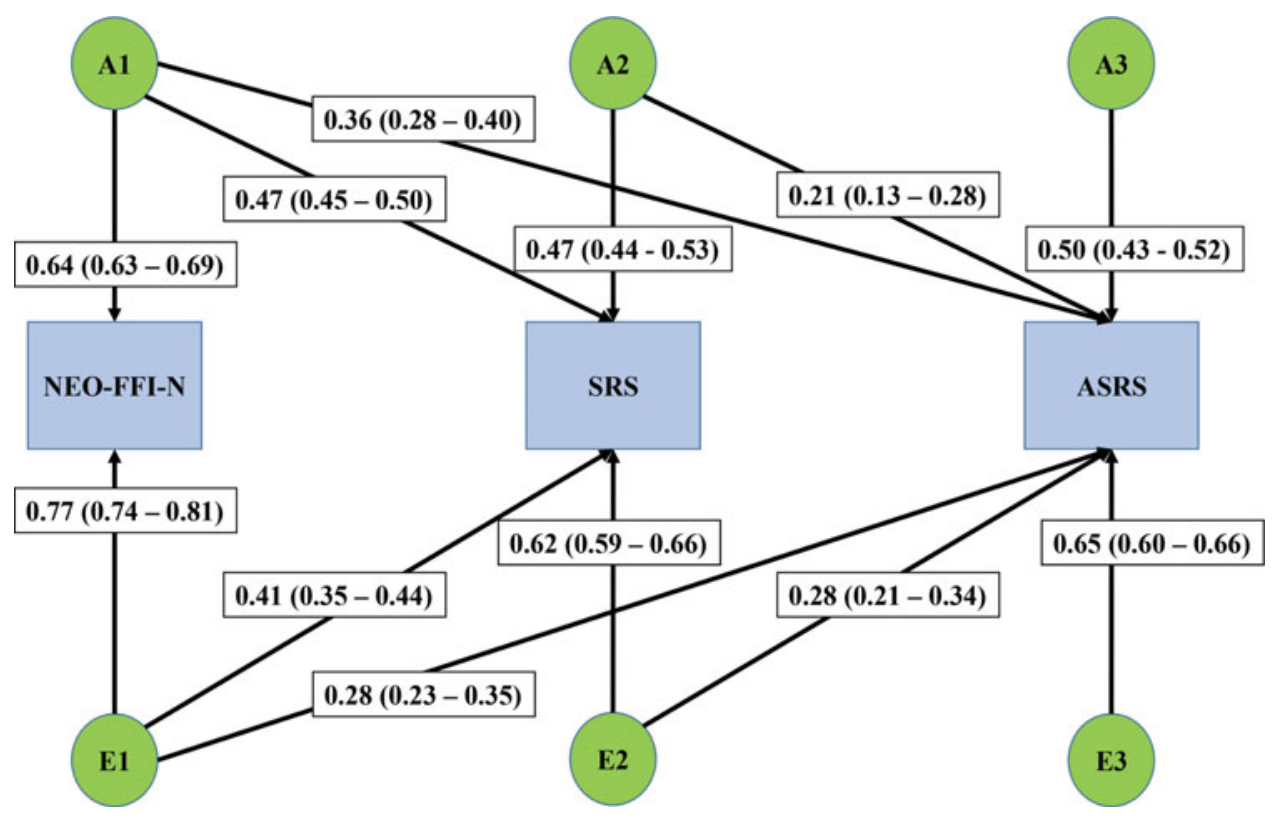

\section{FIGURE 2.}

(Colour online) Path diagram illustrating the trivariate AE Cholesky decomposition model with standardized path coefficients (95\% $\mathrm{Cl}$ ) for neuroticism, autistic, and ADHD traits.

Note: The parameter estimates can be squared to get the percentage of variance of NEO-FFI-N, SRS, and ASRS scores. $\mathrm{A} 1-\mathrm{A} 3=$ additive genetic factors. $\mathrm{E} 1-\mathrm{E} 3=$ unique environmental factors.

factor not shared with NEO-FFI-N and SRS accounted for the remaining genetic variance (25\% of the total variance) in ASRS. On the other hand, all the unique environmental variance (59\% of the total variance) in NEO-FFI-N, 30\% of the unique environmental variance $(17 \%$ of the total variance) in SRS, and $14 \%$ of the unique environmental variance ( $8 \%$ of the total variance) in ASRS could be explained by overlapping unique environmental factor (E1) shared with NEO-FFI-N, SRS, and ASRS. In addition to this, 70\% of the unique environmental variance (38\% of the total vari- ance) in SRS and $14 \%$ of the unique environmental variance ( $8 \%$ of the total variance) in ASRS can be further explained by shared unique environmental factors (E2) between with SRS and ASRS. Over 70\% of the environmental influence (42\% of the total variance) on ASRS scores were not shared with NEO-FFI-N and SRS scores (E3).

As shown in Table 3, the phenotypic correlation between NEO-FFI-N and SRS scores was moderate $\left(r_{\mathrm{p}}=0.62,95 \%\right.$ CI $[0.62,0.64])$. About $49 \%$ of this phenotypic correlation was driven by strong shared genetic influence $\left(r_{\mathrm{g}}=0.71\right.$, 


\section{TABLE 3}

Phenotypic, Additive Genetic and Unique Environmental Correlations $(95 \% \mathrm{Cl})$ Derived From the Trivariate AE Cholesky Decomposition Model of Neuroticism, Autistic and ADHD Traits

\begin{tabular}{|c|c|c|c|}
\hline \multicolumn{4}{|c|}{ Phenotypic correlation $\left(r_{p}\right)$} \\
\hline & NEO-FFI-N & SRS & ASRS \\
\hline NEO-FFI-N & 1 & & \\
\hline SRS & $0.62(0.62-0.64)$ & 1 & \\
\hline ASRS & $0.45(0.43-0.49)$ & $0.56[0.55,0.58]$ & 1 \\
\hline \multicolumn{4}{|c|}{ Genetic correlation $\left(r_{g}\right)$} \\
\hline & NEO-FFI-N & SRS & ASRS \\
\hline NEO-FFI-N & 1 & & \\
\hline SRS & $0.71(0.62-0.74)$ & 1 & \\
\hline ASRS & $0.56(0.53-0.60)$ & $0.63[0.62,0.72]$ & 1 \\
\hline \multicolumn{4}{|c|}{ Unique environmental correlation $\left(r_{\mathrm{e}}\right)$} \\
\hline & NEO-FFI-N & SRS & ASRS \\
\hline NEO-FFI-N & 1 & & \\
\hline SRS & $0.55(0.49-0.61)$ & 1 & \\
\hline ASRS & $0.37(0.30-0.44)$ & $0.51[0.50,0.58]$ & 1 \\
\hline
\end{tabular}

95\% CI $[0.62,0.74])$, with the remaining covariance explained by unique environmental factors $\left(r_{\mathrm{e}}=0.55,95 \%\right.$ CI $[0.49,0.61])$. There was also a weak to moderate phenotypic correlation between NEO-FFI-N and ASRS $\left(r_{\mathrm{p}}=\right.$ $0.45,95 \%$ CI $[0.43,0.49])$ and a moderate phenotypic correlation between SRS and ASRS $\left(r_{\mathrm{p}}=0.56,95 \%\right.$ CI [0.55, $0.58]$ ). Similar to the phenotypic correlation between NEOFFI-N and SRS scores, about $52 \%$ of the correlation between NEO-FFI-N and ASRS was explained by genetic influences $\left(r_{\mathrm{g}}=0.56,95 \%\right.$ CI $\left.[0.53,0.60]\right)$, with the remaining covariance explained by unique environment $\left(r_{\mathrm{e}}=0.37,95 \% \mathrm{CI}\right.$ $[0.30,0.44])$. For the phenotypic correlation between SRS and ASRS, about $49 \%$ of this correlation was accounted for by a moderate degree of overlapping genetic and unique environmental influences $\left(r_{\mathrm{g}}=0.63,95 \%\right.$ CIs [0.62, 0.72]; $r_{\mathrm{e}}=0.51,95 \%$ CI $\left.[0.50,0.58]\right)$.

\section{Discussion}

In the present study, we sought to examine the role of neuroticism in the correlation between ASD and ADHD symptoms by decomposing the variance of ADHD into influences shared with neuroticism and ASD and those shared only with ASD. To the best of our knowledge, this is the first study that has explored the relative contribution of genetic and environmental factors to neuroticism and the causal overlap between ASD and ADHD traits using a twin study. Consistent with previous twin studies in adults (Hansell et al., 2012; Kendler et al., 2007; Lundstrom et al., 2011; Polderman et al., 2014; Reiersen et al., 2008), our heritability estimates for neuroticism, ASD, and ADHD traits were moderate. Moreover, we did not find any significant evidence for common environment effects, genetic dominance, or sex limitation effects. We observed a low correlation between male DZ twins for ADHD traits ( $r=0.02 ; 95 \%$ CI $[-0.21,0.24])$. This finding concurs with previous observations consistent with the presence of sibling contrast effects that might inflate heritability estimates (Reiersen et al.,
2008), although we could not test for sibling contrasts, and dominance or stochastic effects cannot be discarded.

The results of the trivariate twin analyses showed that the genetic factor whose primary effect is on neuroticism (A1 in Figure 2) also has a significant effect on the variance of ASD and ADHD. This factor may reflect a susceptibility to negative emotional states (i.e., anger, fear, anxiety, depression, and stress). It accounted for $41 \%$ of the variance in neuroticism, $22 \%$ of the variance in the ASD traits, and $13 \%$ of the variance in the ADHD traits. The second genetic factor (A2 in Figure 2), which is independent of neuroticism, also accounted for $22 \%$ of the variance in ASD and $4 \%$ of the ADHD. The third genetic factor (A3 in Figure 2) that is specific to ADHD accounted for $25 \%$ of the variance of its variance. Interestingly, the first genetic factor (A1) shared by neuroticism, ASD, and ADHD traits showed a similar degree of genetic contribution to ASD traits as the second genetic factor (A2) that is independent of neuroticism. This factor also showed a higher degree of genetic contribution to ADHD traits, while the second genetic factor (A2) accounted for only $4 \%$ of the variance in ADHD traits, indicating that the common genetic factor is the largest contributor to the comorbidity between the co-occurrence of ASD and ADHD traits.

In contrast, the first unique environmental factor (E1 in Figure 2) shared by neuroticism, ASD, and ADHD traits (A1 in Figure 2) contributed little to the variance (8\%) in ADHD traits while they accounted for $59 \%$ of the variance in neuroticism and $17 \%$ of the variance in ASD traits. The second unique environmental factor (E2 in Figure 2) that is independent of neuroticism, also accounted for $38 \%$ of the variance in ASD traits and $8 \%$ of the variance in ADHD traits. Most of the variance (42\%) of ADHD traits was influenced by unique environmental factor (E3 in Figure 2) specific to ADHD traits. The moderate cross-loading is also consistent with most of $\mathrm{E}$ being measurement error.

The genetic correlation $\left(r_{\mathrm{g}}\right)$ between ASD and ADHD traits reported by previous studies (Reiersen et al., 2008, using a sample partially overlapping with the sample used in the current study (Lundstrom et al., 2011; Polderman et al., $2014)$ were also very similar to ours $\left(r_{\mathrm{g}} \sim 0.60\right)$. Their studies also reported a similar unique environmental correlation between ASD and ADHD traits (approximately half the variance of $r_{\mathrm{p}}$ ). A recent large study (Gale et al, 2016) investigated the evidence of pleiotropy between neuroticism and physical and mental health related traits and found no single nucleotide polymorphism (SNP) genetic correlation between neuroticism and ADHD, indicating insufficient power to detect correlation between common genetic variants or that rare genetic variants shared between family members may contribute more to the variance in neuroticism and ADHD traits.

Apart from a genetic correlation, we found slightly higher unique environmental correlations between ASD and ADHD traits $\left(r_{\mathrm{e}}=0.51 ; 95 \% \mathrm{CI}[0.50,0.58]\right)$ than those 
previously reported unique environmental correlations $\left(r_{\mathrm{e}}\right)$ of 0.22-0.40 between ASD and ADHD traits (Lundstrom et al., 2011; Polderman et al., 2014; Reiersen et al., 2008). We also identified overlapping unique environmental factors related to all traits or only common to ASD and ADHD traits, independent of neuroticism. This suggests that environmental factors might be almost as important as genetic factors to understand the development of comorbidity among neuroticism, ASD, and ADHD traits. It could also reflect the lack of insight, the under-reporting of symptomatology, and the lower accuracy of self-report associated with ADHD (Barkley et al., 2002; Sibley et al., 2012). It is, however, important to note that unique environmental factors may also include measurement error biases.

This study has a number of limitations. First, our measures were based solely on self-reports commonly used in adult population studies. Several lines of evidence reported that adult self-report scales may produce lower heritability estimates in comparison to those typically used in child population studies, such as parental, teacher, or other outside observer ratings (Franke et al., 2012). Second, we used the shortened version of SRS and ASRS questionnaires to minimize subject burden and improve compliance. As a result, autistic and ADHD traits constructed in this present study may be different to those constructed in other studies. Reduction in the number of items used from the SRS is known to diminish the ability to distinguish ASD from general psychopathology (Constantino \& Gruber, 2012; Constantino et al., 2003; Frazier et al., 2014). In addition, our ADHD symptom measure did not include $D S M-I V$ hyperactive symptoms, thus only reflecting inattention and impulsiveness. Third, the distributions of our SRS and ADHD scores were skewed. Derks and colleagues (2004) reported that skewed data could lead to overestimation of non-shared environmental effects and underestimation of shared environmental effects. However, as suggested in their study, we employed the liability threshold model to minimize these parameter estimation biases. Fourth, we obtained our data from a non-clinical population in Australia to increase the representative of our findings and avoid biases inherent in clinic-based studies, such as greater comorbidity and biased gender ratio. However, caution is required as our findings may be different in clinical populations or populations in other countries.

Overall, our findings indicate shared genetic and unique environmental influences among neuroticism, ASD, and ADHD symptoms. These findings not only support evidence of a moderate genetic association between ASD and ADHD traits but indicate that this correlation could be partially explained by etiological factors that these conditions have in common with neuroticism. Our results could reflect that non-disease-specific risk can compound diseasespecific risk in the accumulation of susceptibility to a specific psychiatric disease or variation (Constantino, 2017; Tackett et al., 2013).

\section{Acknowledgments}

We are grateful to the Australian twin participants and project managers for data preparation.

\section{Financial Support}

This research was funded by National Institute on Drug Abuse (NIDA) grant: DA18267 (ML) and by NHMRC grants to NGM. Analysis was partially supported by the NHMRC project grant (1043664) to AJG. The research was facilitated through access to the Australian Twin Registry. Twins Research Australia receives support from the National Health and Medical Research Council through a Centre of Research Excellence Grant, which is administered by the University of Melbourne. AJG is funded by the NHMRC Career Development Fellowship (APP1061922). LCC was supported by the QIMR Berghofer Fellowship.

\section{Conflict of Interest}

None.

\section{References}

Alexander, S. J., \& Harrison, A. G. (2013). Cognitive responses to stress, depression, and anxiety and their relationship to ADHD symptoms in first year psychology students. Journal of Attention Disorders, 17, 29-37.

American Psychiatric Association. (2000). Diagnostic and statistical manual of mental disorders (4th ed., text rev.). Washington, DC: Author.

American Psychiatric Association. (2013). Diagnostic and statistical manual of mental disorders (5th ed.). Washington, DC: Author.

Austin, E. J. (2005). Personality correlates of the broader autism phenotype as assessed by the Autism Spectrum Quotient (AQ). Personality and Individual Differences, 38, 451-460.

Barkley, R. A., Fischer, M., Smallish, L., \& Fletcher, K. (2002). The persistence of attention-deficit/hyperactivity disorder into young adulthood as a function of reporting source and definition of disorder. Journal of abnormal psychology, 111, 279-289.

Boker, S., Neale, M., Maes, H., Wilde, M., Spiegel, M., Brick, T., ... Mehta, P. (2011). OpenMx: An open source extended structural equation modeling framework. Psychometrika, 76, 306-317.

Boomsma, D. I., Saviouk, V., Hottenga, J. J., Distel, M. A., De Moor, M. H., Vink, J. M., ... Willemsen, G. (2010). Genetic epidemiology of attention deficit hyperactivity disorder (ADHD index) in adults. PLoS One, 5, e10621.

Brugha, T. S., McManus, S., Bankart, J., Scott, F., Purdon, S., Smith, J., ... Meltzer, H. (2011). Epidemiology of autism spectrum disorders in adults in the community in England. Archives of General Psychiatry, 68, 459-465. 
Bulik-Sullivan, B., Finucane, H. K., Anttila, V., Gusev, A., Day, F. R., Loh, P.-R., ... Neale, , B. M. (2015). An atlas of genetic correlations across human diseases and traits. Nature Genetics, 47, 1236-1241.

Centers for Disease Control Prevention (CDC). (2014). Prevalence of autism spectrum disorder among children aged 8 years - Autism and developmental disabilities monitoring network, 11 sites, United States, 2010. MMWR Surveillance Summaries, 63, 1-21.

Constantino, J. N., \& Gruber, C. P. (2012). The social responsiveness scale, Second Edition (SRS-2 Manual). Torrance, CA: Western Psychological Services.

Constantino, J. N. (2017). Measurement of autism symptomatology in children with neurodevelopmental impairment. Journal of the American Academy of Child and Adolescent Psychiatry, 56, 354-355.

Constantino, J. N., \& Gruber, C. P. (2005). Social responsiveness scale: Manual. Los Angeles, CA: Western Psychological Services.

Constantino, J. N., Hudziak, J. J., \& Todd, R. D. (2003). Deficits in reciprocal social behavior in male twins: Evidence for a genetically independent domain of psychopathology. Journal of the American Academy of Child and Adolescent Psychiatry, 42, 458-467

Constantino, J. N., Przybeck, T., Friesen, D., \& Todd, R. D. (2000). Reciprocal social behavior in children with and without pervasive developmental disorders. Journal of Developmental and Behavioral Pediatrics, 21, 2-11.

Costa, P. T., \& McCrae, R. R. (1992). Revised Neo Personality Inventory (Neo PI-R) and Neo Five-Factor Inventory (Neo-FFI) (vol. 101). Odessa, FL: Psychological Assessment Resources.

Cross-Disorder Group of the Psychiatric Genomics Consortium. (2013). Genetic relationship between five psychiatric disorders estimated from genome-wide SNPs. Nature Genetics 45, 984-994.

Derks, E. M., Dolan, C. V., \& Boomsma, D. I. (2004). Effects of censoring on parameter estimates and power in genetic modeling. Twin Research, 7, 659-669.

Doshi, J. A., Hodgkins, P., Kahle, J., Sikirica, V., Cangelosi, M. J., Setyawan, J., ... Neumann, P. J. (2012). Economic impact of childhood and adult attention-deficit/hyperactivity disorder in the United States. Journal of the American Academy of Child \& Adolescent Psychiatry, 51, 990-1002.

Duvekot, J., van der Ende, J., Constantino, J. N., Verhulst, F. C., \& Greaves-Lord, K. (2016). Symptoms of autism spectrum disorder and anxiety: Shared familial transmission and cross-assortative mating. Journal of Child Psychology and Psychiatry, 57, 759-769.

Faraone, S. V., Perlis, R. H., Doyle, A. E., Smoller, J. W., Goralnick, J. J., Holmgren, M. A., \& Sklar, P. (2005). Molecular genetics of attention-deficit/hyperactivity disorder. Biological Psychiatry, 57, 1313-1323.

Fayyad, J., De Graaf, R., Kessler, R., Alonso, J., Angermeyer, M., Demyttenaere, K., ... Lépine, J. P. (2007). Crossnational prevalence and correlates of adult attention-deficit hyperactivity disorder. The British Journal of Psychiatry, 190, 402-409.
Franke, B., Faraone, S. V., Asherson, P., Buitelaar, J., Bau, C. H. D., Ramos-Quiroga, J. A., ... Lesch, K. P. (2012). The genetics of attention deficit/hyperactivity disorder in adults, a review. Molecular Psychiatry, 17, 960-987.

Frazier, T. W., Ratliff, K. R., Gruber, C, Zhang, Y, Law, P. A., \& Constantino, J. N. (2014). Confirmatory factor analytic structure and measurement invariance of quantitative autistic traits measured by the social responsiveness scale- 2 . Autism, 18, 31-44.

Gale, C. R., Hagenaars, S. P., Davies, G., Hill, W. D., Liewald, D. C., Cullen, B., ... Smith, D. J. (2016). Pleiotropy between neuroticism and physical and mental health: Findings from 108038 men and women in UK Biobank. Translational Psychiatry, 6, e791.

Gillott, A., \& Standen, P. J. (2007). Levels of anxiety and sources of stress in adults with autism. Journal of Intellectual Disabilities, 11, 359-370.

Gillott, A., Furniss, F., \& Walter, A. (2001). Anxiety in highfunctioning children with autism. Autism, 5, 277-286.

Hansell, N. K., Wright, M. J., Medland, S. E., Davenport, T. A., Wray, N. R., Martin, N. G., \& Hickie, I. B. (2012). Genetic co-morbidity between neuroticism, anxiety/depression and somatic distress in a population sample of adolescent and young adult twins. Psychological medicine, 42, 1249-1260.

Harrison, A. G., Alexander, S. J., \& Armstrong, I. T. (2013). Higher reported levels of depression, stress, and anxiety are associated with increased endorsement of ADHD symptoms by postsecondary students. Canadian Journal of School Psychology, 28, 243-260.

Hoekstra, R. A., Bartels, M., Verweij, C. J., \& Boomsma, D. I. (2007). Heritability of autistic traits in the general population. Archives of Pediatrics \& Adolescent Medicine, 161, 372-377.

Hurtig, T., Kuusikko, S., Mattila, M. L., Haapsamo, H., Ebeling, H., Jussila, K., ... Moilanen, I. (2009). Multi-informant reports of psychiatric symptoms among high-functioning adolescents with Asperger syndrome or autism. Autism, 13, 583-598.

Jacob, C. P., Romanos, J., Dempfle, A., Heine, M., WindemuthKieselbach, C., Kruse, A., ... Brocke, B. (2007). Comorbidity of adult attention-deficit/hyperactivity disorder with focus on personality traits and related disorders in a tertiary referral center. European Archives of Psychiatry and Clinical Neuroscience, 257, 309-317.

Johnston, K., Dittner, A., Bramham, J., Murphy, C., Knight, A., \& Russell, A. (2013). Attention deficit hyperactivity disorder symptoms in adults with autism spectrum disorders. Autism Research, 6, 225-236.

Kendler, K. S., Gardner, C. O., Gatz, M., \& Pedersen, N. L. (2007). The sources of co-morbidity between major depression and generalized anxiety disorder in a Swedish national twin sample. Psychological Medicine, 37, 453-462.

Kessler, R. C., Adler, L., Ames, M., Demler, O., Faraone, S., Hiripi, E. V. A.,... Ustun, T. B. (2005). The World Health Organization Adult ADHD Self-Report Scale (ASRS): A short screening scale for use in the general population. Psychological Medicine, 35, 245-256. 
Knouse, L. E., Traeger, L., O’Cleirigh, C., \& Safren, S. A. (2013). Adult attention deficit hyperactivity disorder symptoms and five-factor model traits in a clinical sample: A structural equation modeling approach. Journal of Nervous and Mental Disease, 201, 848-854.

Lai, M., Lombardo, M. V., \& Baron-Cohen, S. (2014). Autism. Lancet, 383, 896-910.

Larsson, H., Asherson, P., Chang, Z., Ljung, T., Friedrichs, B., Larsson, J. O., \& Lichtenstein, P. (2013). Genetic and environmental influences on adult attention deficit hyperactivity disorder symptoms: A large Swedish populationbased study of twins. Psychological Medicine, 43, 197207.

Leigh, J. P., \& Du, J. (2015). Brief report: Forecasting the economic burden of autism in 2015 and 2025 in the United States. Journal of Autism and Developmental Disorders, 45, 4135-4139.

Lichtenstein, P., Carlström, E., Råstam, M., Gillberg, C., \& Anckarsäter, H. (2010). The genetics of autism spectrum disorders and related neuropsychiatric disorders in childhood. American Journal of Psychiatry, 167, 1357-1363.

Losh, M., Adolphs, R., Poe, M. D., Couture, S., Penn, D., Baranek, G. T., \& Piven, J. (2009). Neuropsychological profile of autism and the broad autism phenotype. Archives of General Psychiatry, 66, 518-526.

Lundström, S., Chang, Z., Kerekes, N., Gumpert, C. H., Råstam, M., Gillberg, C., ... Anckarsäter, H. (2011). Autistic-like traits and their association with mental health problems in two nationwide twin cohorts of children and adults. Psychological Medicine, 41, 2423-2433.

Lynskey, M. T., Heath, A. C., Nelson, E. C., Bucholz, K. K., Madden, P. A. F., Slutske, W. E. E. A., ... Martin, N. G. (2002). Genetic and environmental contributions to cannabis dependence in a national young adult twin sample. Psychological Medicine, 32, 195-207.

Martin, N. G., Eaves, L. J., Kearsey, M. J., \& Davies, P. (1978). The power of the classical twin study. Heredity, 40, 97-116.

Matthews, G., Deary, I. J., \& Whiteman, M. C. (2003) Personality traits (4th ed.). Cambridge, UK: Cambridge University Press.

Michielsen, M., Comijs, H. C., Semeijn, E. J., Beekman, A. T., Deeg, D. J., \& Kooij, J. S. (2014). Attention deficit hyperactivity disorder and personality characteristics in older adults in the general Dutch population. American Journal of Geriatric Psychiatry, 22, 1623-1632.

Musser, E. D., Hawkey, E., Kachan-Liu, S.S., Lees, P., Roullet, J. B., Goddard, K., ... Nigg, J. T. (2014). Shared familial transmission of autism spectrum and attentiondeficit/hyperactivity disorders. Journal of Child Psychology and Psychiatry and Allied Disciplines, 55, 819-827.

Neale, M., \& Cardon, L. (1992). Methodology for genetic studies of twins and families. Dordrecht, The Netherlands: Kluwer Academic Publishers.

Nigg, J. T., John, O. P., Blaskey, L. G., Huang-Pollock, C. L., Willicut, E. G., Hinshaw, S. P., \& Pennington, B. (2002). Big five dimensions and ADHD symptoms: Links between personality traits and clinical symptoms. Journal of Personality and Social Psychology, 83, 451.
Parker, J. D., Majeski, S. A., \& Collin, V. T. (2004). ADHD symptoms and personality: Relationships with the fivefactor model. Personality and Individual Differences, 36, 977-987.

Polderman, T. J. C., Hoekstra, R. A., Posthuma, D., \& Larsson, H. (2014). The co-occurrence of autistic and ADHD dimensions in adults: An etiological study in 17,770 twins. Translational Psychiatry, 4, e435.

Polderman, T. J. C., Hoekstra, R. A., Vinkhuyzen, A. A. E., Sullivan, P. F., van der Sluis, S., \& Posthuma, D. (2013). Attentional switching forms a genetic link between attention problems and autistic traits in adults. Psychological Medicine, 43, 1985-1996.

Rao, P. A., \& Landa, R. J. (2014). Association between severity of behavioral phenotype and comorbid attention deficit hyperactivity disorder symptoms in children with autism spectrum disorders. Autism, 18, 272-280.

Reiersen, A. M., Constantino, J. N., Grimmer, M., Martin, N. G., \& Todd, R. D. (2008). Evidence for shared genetic influences on self-reported ADHD and autistic symptoms in young adult Australian twins. Twin Research and Human Genetics, 11, 579-585.

Rommelse, N. N., Franke, B., Geurts, H. M., Hartman, C. A., \& Buitelaar, J. K. (2010). Shared heritability of attention-deficit/hyperactivity disorder and autism spectrum disorder. European Child \& Adolescent Psychiatry, 19, 281-295.

Schriber, R. A., Robins, R. W., \& Solomon, M. (2014). Personality and self-insight in individuals with autism spectrum disorder. Journal of Personality and Social Psychology, 106, 112-130.

Schwartzman, B. C., Wood, J. J., \& Kapp, S. K. (2016). Can the five factor model of personality account for the variability of autism symptom expression? Multivariate approaches to behavioral phenotyping in adult autism spectrum disorder. Journal of Autism and Developmental Disorders, 46, 253272.

Sibley, M. H., Pelham, W. E., Molina, B. S. G., Gnagy, E. M., Waxmonsky, J. G., Waschbusch, D. A., ... Kuriyan, A. B. (2012). When diagnosing ADHD in young adults emphasize informant reports, DSM items, and impairment. Journal of Consulting and Clinical Psychology, 80, 1052-1061.

Tackett, J. L., Lahey, B. B., Hulle, C. V., Waldman, I., Krueger, R. F., \& Rathouz, P. J. (2013). Common genetic influences on negative emotionality and a general psychopathology factor in childhood and adolescence. Journal of Abnormal Psychology, 122, 1142-1153.

Todd, R. D., Huang, H., Todorov, A. A., Neuman, R. J., Reiersen, A. M., Henderson, C. A., \& Reich, W. C. (2008). Predictors of stability of attention-deficit/hyperactivity disorder subtypes from childhood to young adulthood. Journal of the American Academy of Child \& Adolescent Psychiatry, 47, 76-85.

Van den Berg, S. M., Willemsen, G., de Geus, E. J., \& Boomsma, D. I. (2006). Genetic etiology of stability of attention problems in young adulthood. American Journal of Medical Genetics Part B: Neuropsychiatric Genetics, 141, 55-60. 
van Steijn, D. J., Richards, J. S., Oerlemans, A. M., de Ruiter, S. W., van Aken, M. A., Franke, B., ... Rommelse, N. N. (2012). The co-occurrence of autism spectrum disorder and attention-deficit/hyperactivity disorder symptoms in parents of children with ASD or ASD with ADHD. Journal of Child Psychology and Psychiatry and Allied Disciplines, 53, 954-963.

Visser, S. N., Danielson, M. L., Bitsko, R. H., Holbrook, J. R., Kogan, M. D., Ghandour, R. M., ... Blumberg, S. J. (2014). Trends in the parent-report of health care providerdiagnosed and medicated attention-deficit/hyperactivity disorder: United States, 2003-2011. Journal of the American Academy of Child \& Adolescent Psychiatry, 53(1), 34-46.

Wakabayashi, A., Baron-Cohen, S., \& Wheelwright, S. (2006). Are autistic traits an independent personality dimension? A study of the Autism-Spectrum Quotient (AQ) and the NEO-PI-R. Personality and Individual Differences, 41, 873883.

Zuckerman, M., \& Stelmack, R. M. (2004). On the psychobiology of personality: Essays in honor of Marvin Zuckerman. Oxford, UK: Elsevier. 